\title{
Análise da interferência de nuvens na classificação de séries temporais MODIS-NDVI na região da Amazônia, municícpio de Capixaba, Acre
}

\author{
Cloud interference analysis in the classification of MODIS-NDVI \\ temporal series in Amazon region, municipality of Capixaba, Acre - \\ Brazil
}

\author{
Cristiane Batista Salgado ${ }^{1}$ \\ Osmar Abílio de Carvalho Júnior ${ }^{2}$ \\ Roberto Arnaldo Trancoso Gomes ${ }^{3}$ \\ Renato Fontes Guimarães ${ }^{4}$
}

\begin{abstract}
Resumo
Este trabalho teve como objetivo analisar classificações de séries temporais MODIS-NDVI, com 3 diferentes algoritmos, buscando identificar a quantidade ideal de imagens para estudos em ambientes com altas taxas de nebulosidade. O recorte espacial utilizado para o estudo foi o município de Capixaba, localizado no Estado do Acre na região amazônica. Para cada imagem NDVI, construiu-se uma máscara de nuvem. Esta máscara permitiu organizar o cubo temporal por quantitativo de cobertura de nuvem. Assim, testou-se o impacto da eliminação das imagens com alta nebulosidade para a classificação da série. A cada corte, o cubo temporal foi refeito, avaliando resultados para um novo conjunto de bandas. Para a análise de acurácia, adotou-se o coeficiente Kappa. Ao todo foram feitas 84 classificações. Foram testados 3 algoritmos de classificação (Mínima Distância, Spectral Angle Maper e Spectral Correlation Mapper) e 4 diferentes interações entre classes e amostras. Observou-se que, ao longo do período analisado, aproximadamente $80 \%$ das imagens apresentaram cobertura de nuvens acima de $90 \%$. Os testes mostraram que a retirada das imagens com nuvem, aumentou a qualidade da classificação, sendo que os melhores resultados foram encontrados em cubos pequenos (10 a 35 imagens) e com baixa cobertura de nuvem (0 a 6 \%). O algoritmo Mínima Distância apresentou o menor coeficiente de variação dentre os resultados encontrados, mostrando menor sensibilidade à presença de nuvens.
\end{abstract}

Palavras-chave: cobertura de nuvem; series temporais; Amazônia

\footnotetext{
Abstract

This research aimed to analyze MODIS-NDVI time series classifications, with three different algorithms, seeking to identify the ideal amount of

${ }^{1}$ Instituto Federal de Brasília, Brasília, Distrito Federal, Brasil. cristiane.salgado@ifb.edu.br

2 Universidade de Brasília, Brasília, Distrito Federal. osmarjr@unb.br

${ }^{3}$ Universidade de Brasília, Brasília, Distrito Federal. $\underline{\text { robertogomes@unb.br }}$

${ }^{4}$ Universidade de Brasília, Brasília, Distrito Federal. renatofg@unb.br

Artigo recebido em: 18/02/2019. Aceito para publicação em: 12/08/2019.
} 
images for studies in environments with high cloudiness rates. The spatial cut used for the study was the municipality of Capixaba, located in the state of Acre in the Amazon region. For each NDVI image, a cloud mask was constructed. This mask allowed to organize the temporal cube by cloud coverage quantity. Thus, the impact of eliminating high cloud images for the series classification was tested. At each cut, the temporal cube was redone, evaluating results for a new set of bands. For the accuracy analysis, the Kappa coefficient was adopted. A total of 84 classifications were made. Three classification algorithms (Minimum Distance, Spectral Angle Mapper and Spectral Correlation Mapper) and 4 different interactions between classes and samples were tested. Over the period analyzed, approximately $80 \%$ of images showed cloud cover above $90 \%$. The tests showed that the removal of the images with cloud increased the quality of the classification, and the best results were found in small cubes (10 to 35 images) and with low cloud cover ( 0 to $\sim 6 \%$ ). The Minimum Distance algorithm presented the lowest coefficient of variation, showing a lower sensitivity to the presence of clouds.

Keywords: Cloud cover. Time series. Amazon

\section{Introdução}

A análise espacial de dados geográficos permite mensurar propriedades, dinâmicas e relacionamentos, considerando a localização espacial dos fenômenos (CÂMARA et al., 2002). As geotecnologias aplicadas às análises espaciais trouxeram a possibilidade de representação e monitoramento das diversas dinâmicas da superfície terreste, acelerando a disponibilidade de dados no tempo (FITZ, 2008). Através delas torna-se possível observar as transformações no uso e ocupação da terra, na dinâmica hidrográfica, no crescimento urbano, no monitoramento dos cultivos agrícolas, das queimadas, do desflorestamento, além de gerar por exemplo, índices de vegetação (HUETE et al., 2002)

A disponibilidade de séries temporais totalmente livres de ruídos está relacionada às condições climáticas das regiões, o que limita as informações disponíveis para algumas áreas do globo (BRASWELL et al., 2003). O acompanhamento de curvas temporais dos diversos alvos da superfície terrestre, permite diferenciá-los nos mapeamentos, além de permitir a gestão dos recursos ambientais e da produção agrícola (MASSEY et al., 2017). 
$\mathrm{Na}$ Amazônia, esse tipo de análise é dificultada pela frequente cobertura de nuvens, o que afeta diretamente as imagens dos sensores opticos (ASNER, 2001). Embora, o sensoriamento remoto por satélites forneça um meio viável de observar a Amazônia de forma espacialmente abrangente e temporalmente frequente, a avaliação óptica das áreas tropicais fica comprometida pela alta concentração de aerossóis (queima de biomassa) e nuvens (ALI; DE BIE; SKIDMORE, 2013; ARTAXO et al., 2013; ASNER, 2001; CHEN et al., 2004; HILKER et al., 2015; MARTINS et al., 2018).

Os sensores de alta resolução temporal aumentam a probabilidade da aquisição de imagens livres de ruídos ao longo do ano para os estudos que envolvem a detecção de mudança. O sensor Moderate Resolution Imaging Spectroradiometer (MODIS) disponibiliza imagens a cada 01 ou 02 dias, numa resolução espacial de 250m (LATORRE et al., 2003; SHELDON; XIAO; BIRADAR, 2012). A sua alta resolução temporal pode suprir lacunas temporais de outros sensores (causadas pela cobertura de nuvens), como por exemplo, o sensor TM, disponibilizando um número maior de informações para diversos estudos (LI et al., 2018).

A maior parte das imagens livres de nuvem disponíveis para a Amazônia encontra-se no período de estiagem, sobretudo de Julho a Setembro. No período chuvoso há um aumento da cobertura de nuvem (MARTINS et al., 2018), impactando diretamente as análises de séries temporais. O presente trabalho objetiva avaliar as classificações de uso e ocupação do solo em dados MODIS considerando a quantidade ideal de imagens a ser utilizadas numa série temporal, de forma a garantir os melhores resultados. 


\section{Material e métodos}

As etapas metodológicas do trabalho podem ser organizadas em três processamentos (Figura1). A primeira etapa diz respeito a contrução dos cubos temporais do NDVI (Normalized Difference Vegetation Index) e da máscara de nuvem. A segunda etapa diz respeito da escolha das amostras e classes de trabalho. A terceira etapa se refere aos testes de classificação executadas no programa Abilius ${ }^{\circledR}$. Estas etapas serão melhor descritas abaixo.

Figura 1 - Fluxograma das etapas metodológicas do trabalho considerando os processos de processamento de dados 1 (cubos temporais), 2(amostras), e 3(classificações).

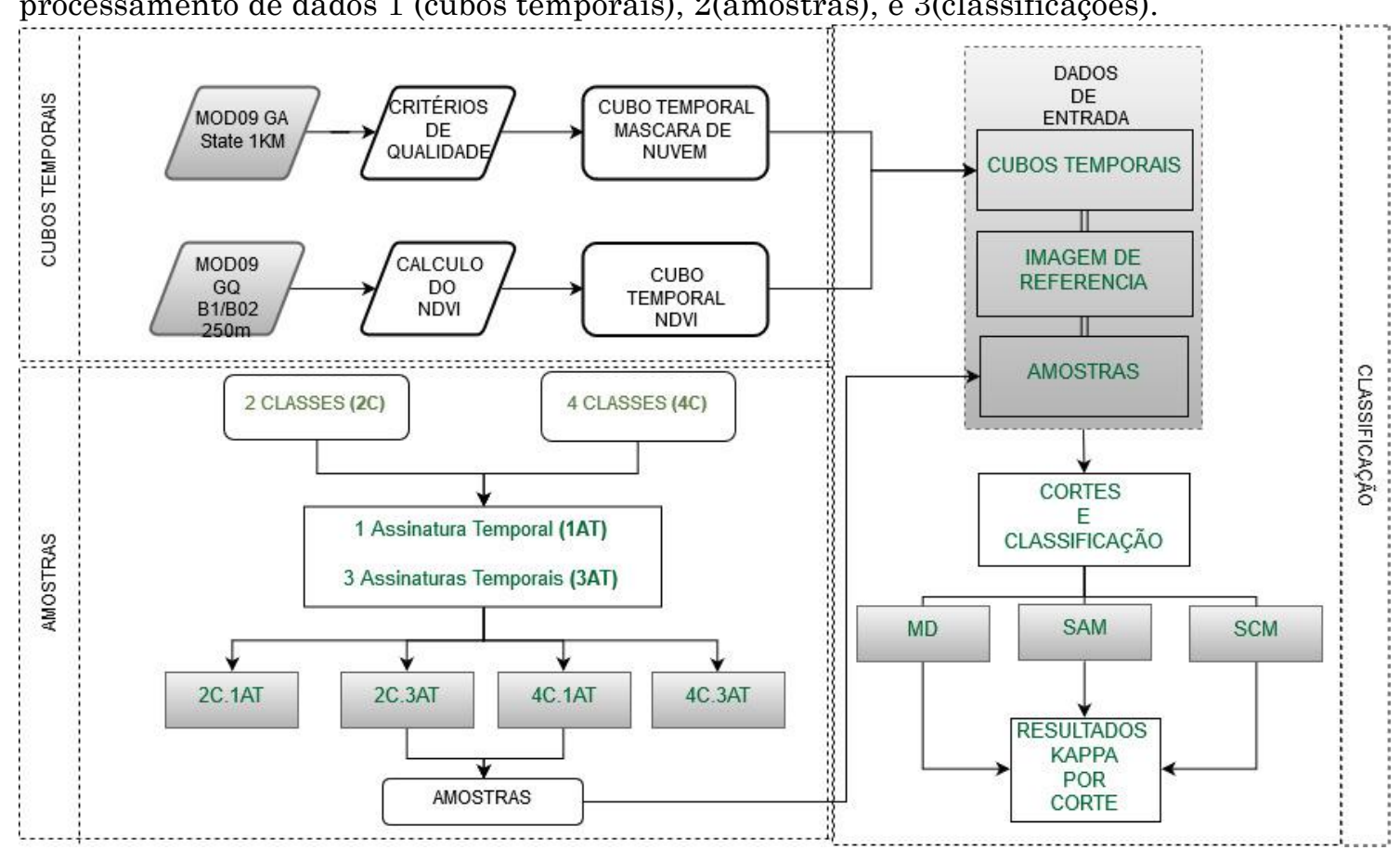

Org.: dos Autores, 2018.

\section{Área de estudo}

A área de estudo é um recorte que envolve o município de Capixaba e uma parte dos municípios de Plácido de Castro, Senador Guiomar, Xapuri e Rio Branco, no Estado do Acre (Figura 2). Os municípios de Capixaba, Senador Guiomard e Plácido de Castro concentram plantações de cana-de- 
açúcar, à margem da BR-317, áreas próximas à Usina Álcool Verde. O recorte foi escolhido para que essa dinâmica pudesse ser considerada nos testes de classificação.

O município de Capixaba possui $47,83 \%$ de sua cobertura florestal preservada, Senador Guiomar 28,01\% e Plácido de Castro 25.98\% (INPE, 2017). A atividade agropecuária é parte significativa do PIB dos municípios, sendo 45,02\% em Capixaba, 31,07\% em Senador Guiomar e 28,75\% em Plácido de Castro, considerando o ano de 2015 (IBGE, 2016). O efetivo bovino do município de Capixaba é de 155.881 cabeças e os principais cultivos em 2015 foram cana-de-açúcar (174.180 toneladas em 2.903 ha), milho (5.396 toneladas em 1978 ha) e mandioca (31.207 toneladas em 1.172 ha) (IBGE, 2016). Ainda em 2015, os municípios vizinhos, Senador Guiomar e Plácido de Castro, contribuíram com a produção de cana-de-açúcar, pela proximidade com a usina Álcool Verde, cujo total foi de 4.785 e 525 toneladas, respectivamente (IBGE, 2016).

Figura 2 - Mapa de localização da área de estudo.
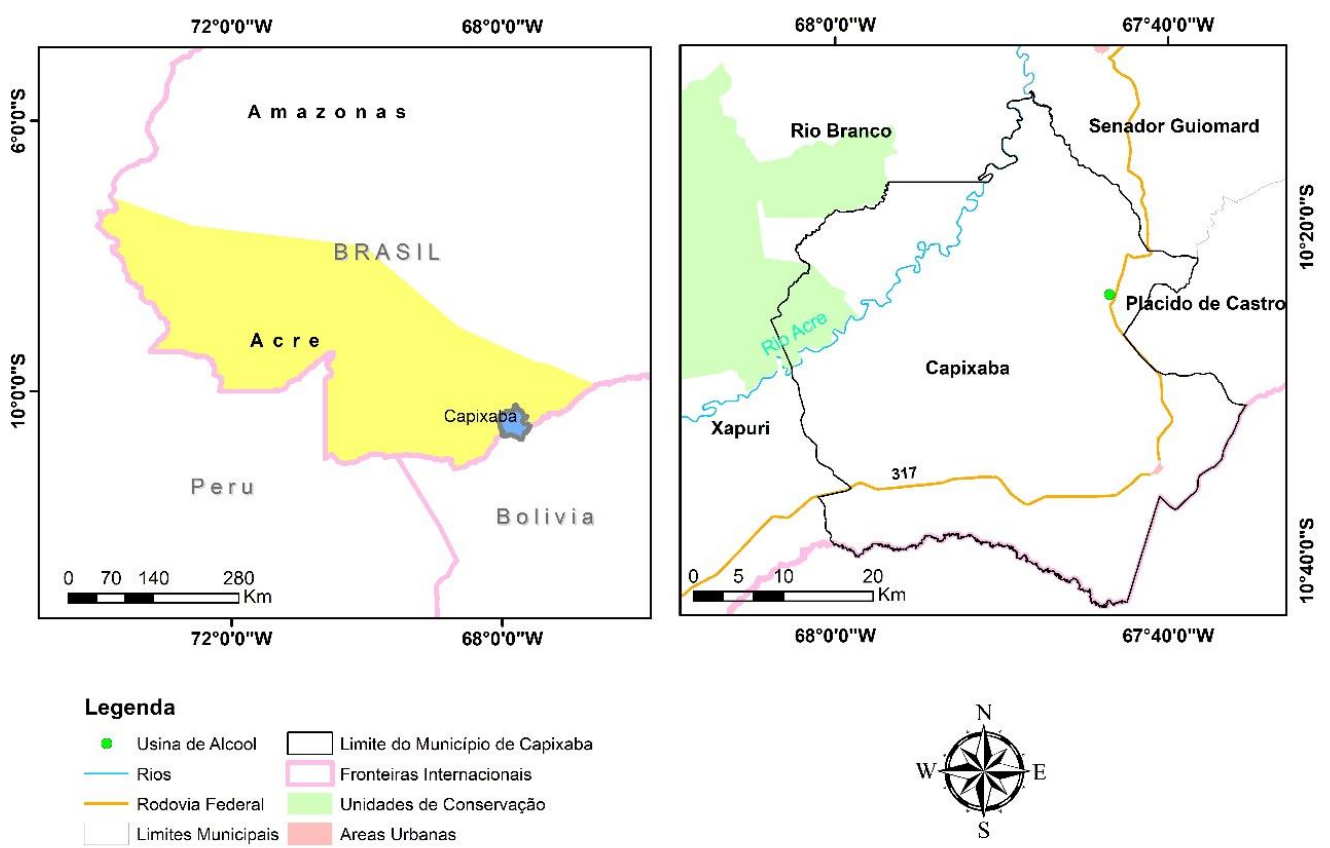

Fonte: Dados digitais do Zoneamento Ecológico Econômico do Estado do Acre.

Org.: dos Autores, 2018. 


\section{Dados do sensor MODIS}

O sensor MODIS (Moderate Imaging Spectroradiometer) está em operação desde 2000 à bordo da plataforma Terra e desde 2002 na plataforma Aqua, fornecendo imagens globais duas vezes ao dia. Sua resolução espacial varia de $250 \mathrm{~m}$ a $1 \mathrm{~km}$, possuindo 36 bandas espectrais. Além disso os dados podem ser acessados gratuitamente através do site Earth Data/NASA (Nacional Aeronautics and Space Adminstrate).

Dois produtos MODIS foram utilizados: (a) produto MOD09GQ com dados diários para a área de interesse, contendo as bandas 1 e 2 (red e infrared) na resolução de $250 \mathrm{~m}$, que permite gerar o Índice de Vegetação da Diferença Normalizada (NDVI); e (b) produto MOD09GA contendo imagens diárias com resolução de $1 \mathrm{~km}$ sobre a qualidade das imagens, possibilitando criar a máscara de nuvem ( VERMOTE, 2011; DAAC; FALLS, 2014)

O NDVI se mostra sensível a clorofila, além de minimizar as interferências relativas às variações provenientes do ângulo solar e dos efeitos atmosféricos (SANTANA et al., 2010). O cálculo desse índice é dado pela seguinte expressão:

$$
N D V I=\frac{\rho \mathrm{IVP}-\rho \mathrm{V}}{\rho \mathrm{IVP}+\rho \mathrm{V}}
$$

onde, $\rho I V P$ é o valor da reflectância no infravermelho próximo (800-1100 nm) e $\rho V$ é a reflectância na faixa do vermelho (600-700 nm).

$\mathrm{Na}$ elaboração das máscaras de nuvem, os seguintes critérios foram escolhidos: cloud state (clear), aerosol quantity (climatology and low) e cirrus detect (none and small). A máscara foi reamostrada para 250 metros para que as imagens NDVI fossem compatíveis. Ambos os produtos foram adquiridos para o período de agosto de 2014 a julho de 2015. 
É comum a filtragem das séries temporais do sensor MODIS, como por exemplo, filtro de mediana, para eliminação de ruídos (DE CARVALHO JUNIOR et al., 2008; DE CARVALHO JÚNIOR et al., 2012). Neste trabalho, optou-se pela não filtragem dos dados para que se mantivesse a relação exata entre o pixel da máscara e o pixel da imagem NDVI, uma vez que a filtragem poderia alterar os seus valores.

\section{Classificação Temporal}

Na presente pesquisa, os testes de classificação temporal consideraram 2 classes (floresta e não floresta) e 4 classes de uso e cobertura da terra (floresta, pasto, cana-de-açúcar e água). Além disso, as assinaturas temporais utilizadas no treinamento foram obtidas por dois procedimentos: (a) conversão das classes do mapa de referência em regiões de interesse no cubo NDVI, extraindo a assinatura temporal média de cada classe; e (b) as mesmas regiões de interesse foram trabalhadas no módulo "N-Dimensional Visualiser" do programa Envi 5.0 para a seleção de três assinaturas temporais que representassem as principais variações da amostra (ABADE et al., 2015). Logo, executou-se 4 combinações para os testes de classificação: (a) 4 classes com 3 assinaturas temporais (4C3AT); (b) 4 classes com 1 assinatura temporal (4C1AT); (c) 2 classes com 3 assinaturas temporais (2C.3AT), e (d) 2 classes com 1 assinatura temporal (2C1AT) (Figura 1).

Desenvolveu-se um módulo específico do programa Abilius ${ }^{\circledR}$, que trabalha concomitantemente com a máscara de nuvens e o cubo NDVI, bem como com as assinaturas temporais, a classificação e a acurácia dos dados (Figura 1). A princípio o algoritmo calcula a porcentagem de nuvem em cada cena NDVI de acordo com os dados da máscara de nuvem. Em seguida, o cubo NDVI é ordenado automaticamente de maior para menor porcentagem de cobertura de nuvens. O método realiza uma série de classificações, onde a primeira classificação (ponto 0) considera toda a série temporal, enquanto as 
demais realizam cortes subsequentes das imagens com maior interferência de nuvens em uma sequência linear, considerando um intervalo de imagens determinado pelo usuário. O programa permite a escolha de três classificadores: Mínima Distância, Spectral Angle Mapper (SAM) e Spectral Correlation Mapper (SCM), que foram avaliados nesse trabalho. As diferentes sequências de classificações temporais são comparados com o mapa de referência, utilizando o coeficiente Kappa, amplamente utilizado na análise de acurácia de classificações de imagens de sensoriamento remoto ( MELLO et al., 2011; CHEN et al., 2015). O coeficiente Kappa usa uma técnica multivariada discreta que considera todos os elementos da matriz de confusão (CONGALTONS, 1991; FONSECA, 2000). Quanto aos níveis mínimos aceitáveis desse coeficiente, eles podem variar de péssimo a excelente (LANDIS; KOCH, 1977), com valores entre 0 e 1 (Tabela 1).

Tabela 1 - Níveis de desempenho do índice Kappa.

\begin{tabular}{cc}
\hline Índice Kappa(k) & Classes de desempenho \\
\hline$<0$ & Péssimo \\
$0<\mathrm{k} \leq 0,2$ & Ruim \\
$0,2<\mathrm{k} \leq 0,4$ & Razoável \\
$0,4<\mathrm{k} \leq 0,6$ & Bom \\
$0,6<\mathrm{k} \leq 0,8$ & Muito bom \\
$0,8<\mathrm{k} \leq 1,0$ & Excelente \\
\hline
\end{tabular}

Fonte: Landis, Koch, 1997. Adpatado pelos autores, 2018.

O mapa de referência foi uma atualização do mapa de uso e cobertura do solo de 2011 (desenvolvido pelo Instituto Acre do Meio Ambiente usando imagens do Rapdeye) a partir da interpretação visual usando imagens do Landsat 8 (cena 002/67, agosto de 2015). As áreas de cana-de-açúcar foram levantadas em trabalho de campo realizado durante outubro de 2015 (Figura 3). Realizou-se um total de 84 classificações para cada algoritmo (MD, SAM, SCM) e para cada combinação estabelecida (4C3AT / 4C1AT / 2C3AT / 2C1AT). 
Figura 3. (A) Mapa de referência utilizado na validação das classificações e (B) Imagem Landsat utilizada na atualização do mapa - composição 654, 06 de agosto de 2015.

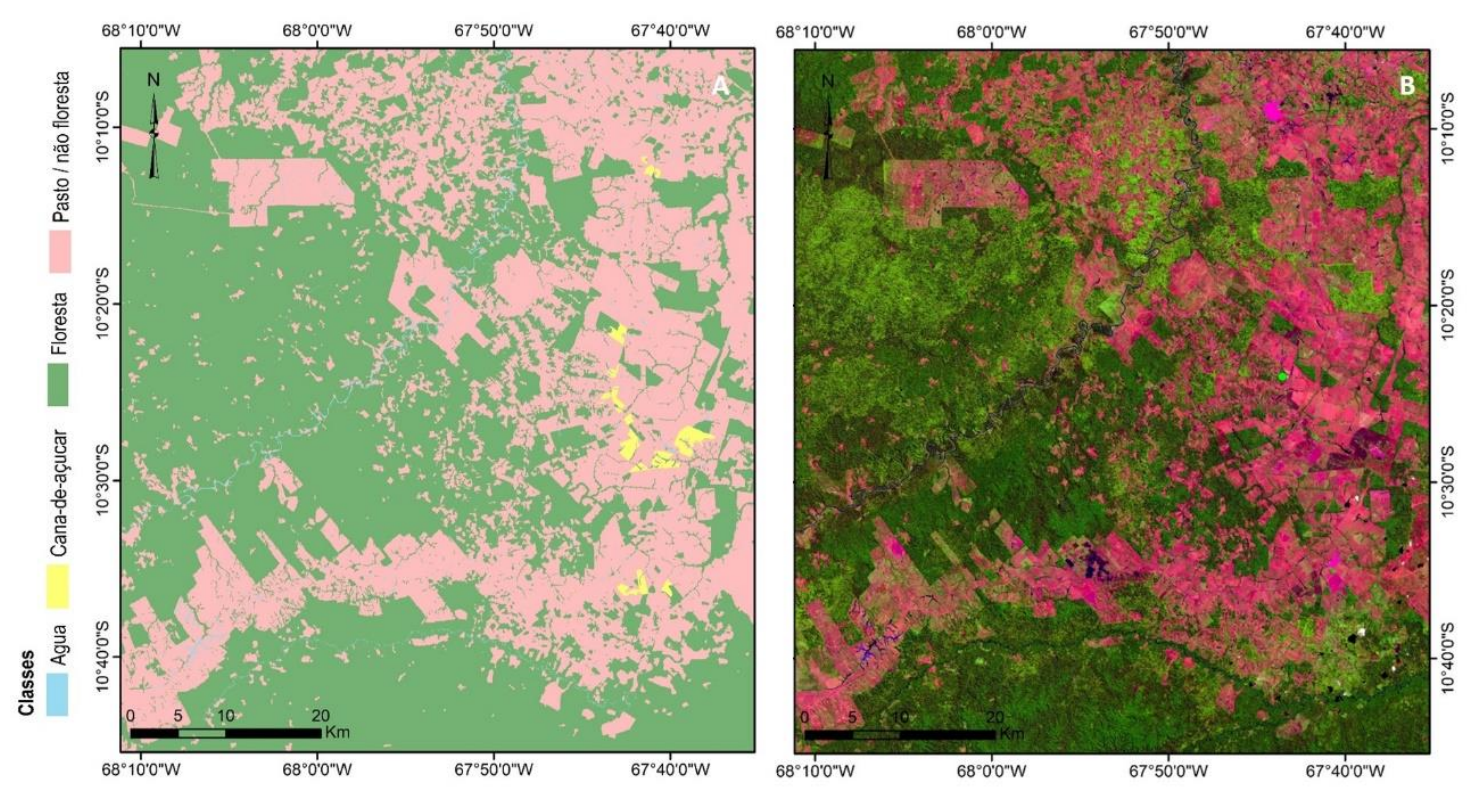

Fonte: Instituto de Meio Ambiente do Acre- IMAC. Serviço Geológico do Estados Unidos - USGS. Org.: dos Autores, 2018.

\section{Resultados}

Conforme o esperado, as sequências temporais do coeficiente Kappa demonstraram uma expressiva subida inicial com a desconsideração das imagens com maior cobertura de nuvem até atingir patamares de estabilidade, havendo no final uma queda gradual com a diminuição das informações temporais (Figura 4). As classificações com duas classes (floresta e não floresta) tiveram melhores resultados em relação às classificações com quatro classes, demonstrando a dificuldade da separação das classes contidas na classe não floresta. Além disso, na maioria dos testes os modelos constituídos com apenas uma assinatura temporal média tiveram melhores resultados do que com três assinaturas com variações da mesma amostra.

Dentre as classificações, o modelo $2 \mathrm{C} 1 \mathrm{AT}$ usando o algoritmo $\mathrm{MD}$ obteve o maior valor de coeficiente Kappa com 0,755 (nível de desempenho muito bom), selecionando 34 imagens do cubo temporal (corte 56) com 
cobertura de nuvem variando entre 0 e 5,62\%. Nessa combinação foi possível observar um comportamento diferenciado dos demais modelos em que a partir do corte 1 , quando foram eliminadas as imagens com $90 \%$ ou mais de nuvem, o valor do coeficiente Kappa manteve-se acima de 0,7, mostrando a eficiência do classificador em diferentes condições (Figura 4). O modelo 2C3AT usando o algoritmo MD atingiu um coeficiente Kappa de 0,632, com a seleção de 20 imagens no cubo temporal (corte 70). No entanto, o melhor coeficiente Kappa nas combinações com 4 classes foi obtido quando utilizou-se 3 assinaturas temporais $(0,544)$ em vez de 1 assinatura $(0,532)$, selecionando o corte $78 \mathrm{com}$ apenas 12 imagens no cubo (Tabela 2). Esse foi o único modelo onde o emprego de três assinaturas temporais superou a de uma assinatura considerando as demais variáveis de combinações iguais.

Os algoritmos SAM e SCM tiveram comportamento semelhantes em relação aos cortes e combinações (Figura 4). Verifica-se um incremento do coeficiente Kappa a cada corte, com pequenas variações $(0,01$ a 0,02). É importante destacar que a partir do corte 35 o coeficiente Kappa ganha relativa estabilidade para as combinações que utilizaram 2 classes e a partir do corte 38 para as combinações que utilizaram 4 classes. A composição 2C1AT obteve o melhor coeficiente Kappa de 0,738, encontrado no corte 59 com um série temporal de 31 imagens com cobertura de nuvens variando entre 0 e 3,45\% (Tabela 2). Os classificadores SAM e SCM conseguem superar o classificador MD no modelo 2C3AT e 4C1AT (Figura 5). No modelo 4C1AT os classificadores SAM e SCM aumentam a cada corte, superando o classificador $\mathrm{MD}$ que se mantêm praticamente estável ao longo dos cortes (coeficiente de variação de 5,46). 
Figura 4 - Os valores de coeficiente Kappa resultantes da classificação com os algoritmos MD, SAM e SCM, por corte, por combinação: 4C3AT; 4C1AT; 2C3AT; 2C1AT.

MD

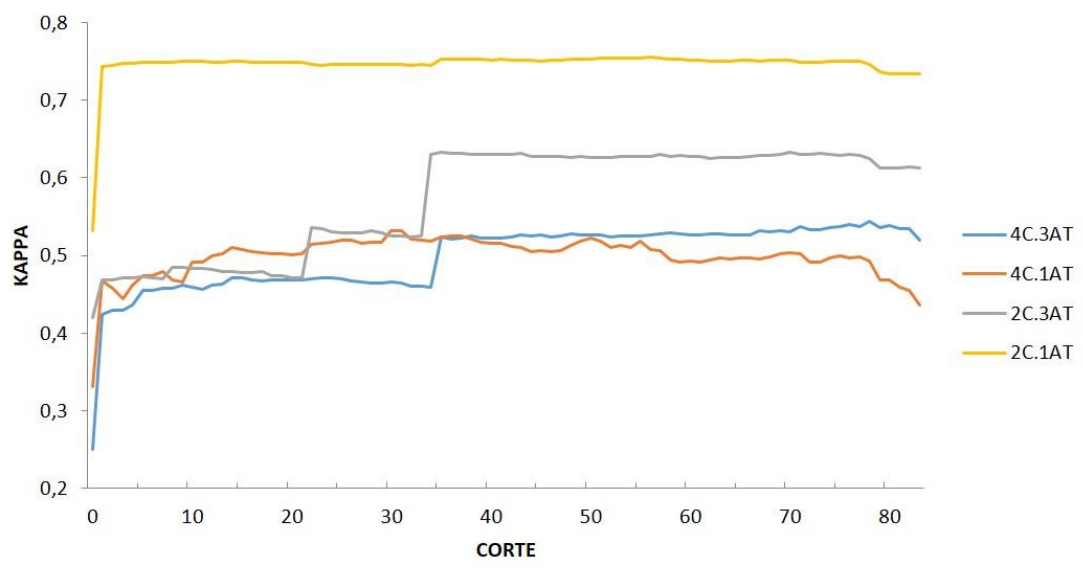

SAM

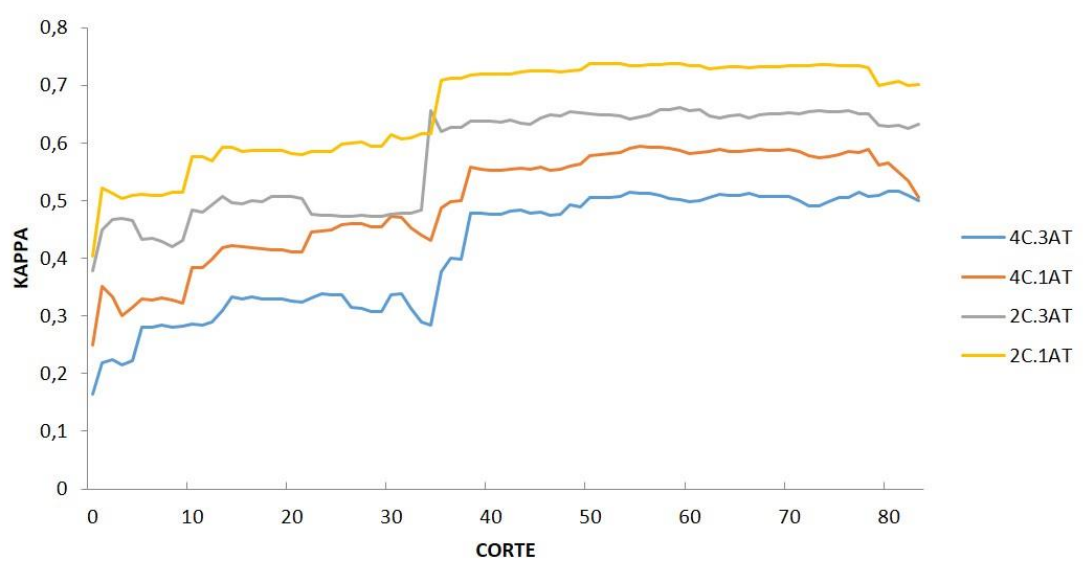

SCM

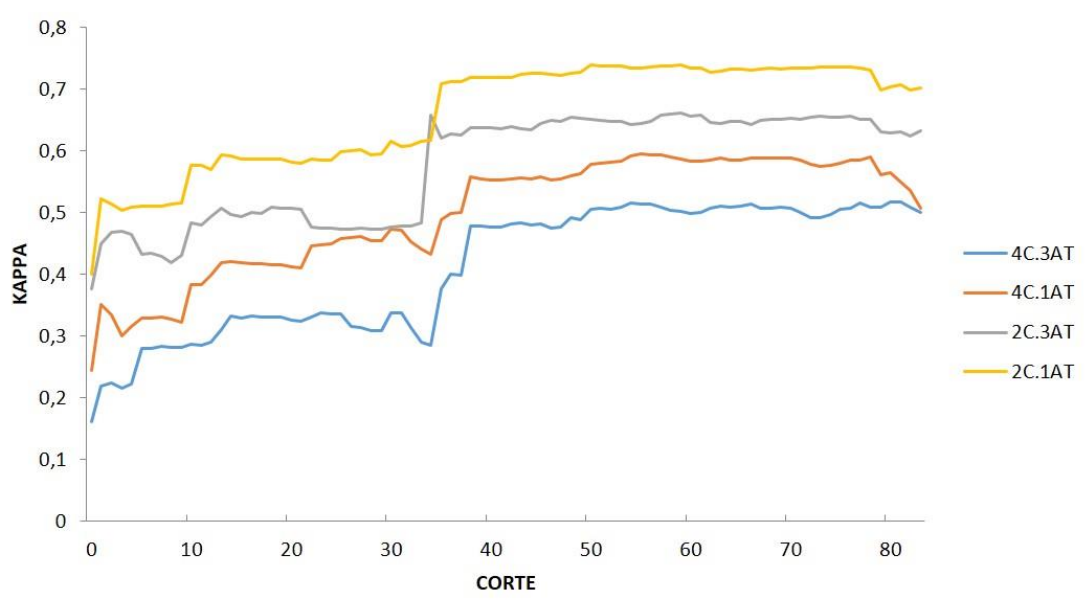

Org.: dos Autores, 2018. 
Figura 5 - Comparação dos classificadores SAM, SCM e MD entre as combinações de 4 e 2 classes

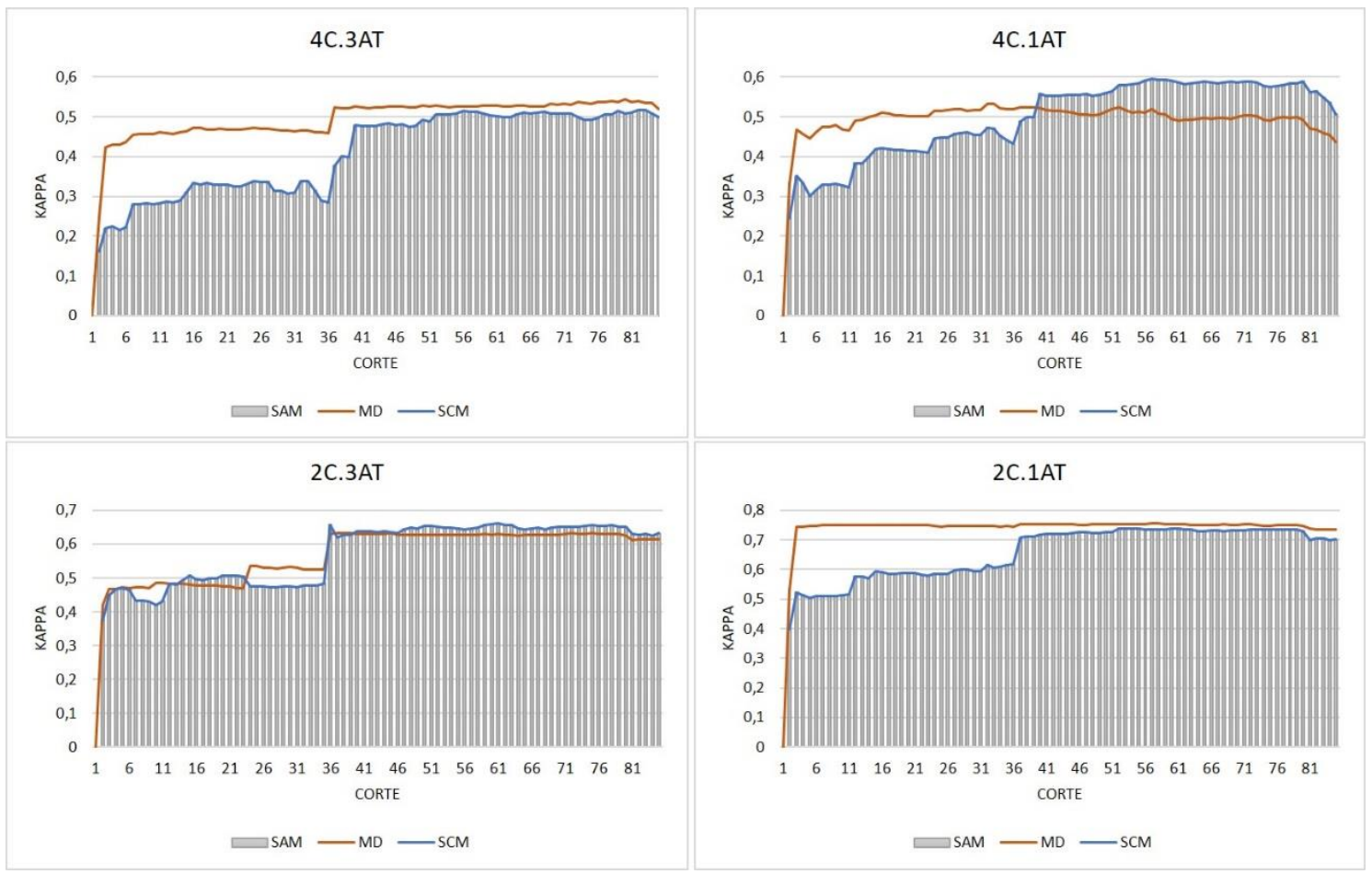

Org.: dos Autores, 2018.

Tabela 2 - Melhor coeficiente Kappa por algoritmo (MD, SAM, SCM) e combinação.

\begin{tabular}{ccccc}
\hline Análise (a) & MC.3AT & 4C.1AT & 2C.3AT & 2C.1AT \\
Maior Kappa & 0,544 & 0,532 & 0,632 & 0,755 \\
Número do Corte & 78 & 31 & 70 & 56 \\
Número de imagens do cubo* & 12 & 59 & 20 & 34 \\
\% de cobertura de nuvem ** & 0 & 54,13 & 0,07 & 5,62 \\
Kappa no corte 0 & 0,24 & 0,33 & 0,41 & 0,53 \\
Análise (b) & & SAM/SCM & 2C.1AT \\
Maior Kappa SAM & $\mathbf{4 C . 3 A T}$ & $\mathbf{4 C . 1 A T}$ & $\mathbf{2 C . 3 A T}$ & 0,738362 \\
Maior Kappa SCM & 0,516971 & 0,594528 & 0,661072 & 0,738364 \\
Número do corte & 0,516997 & 0,594627 & 0,661100 & 59 \\
Número de imagens do cubo* & 80 & 55 & 59 & 31 \\
\% de cobertura de nuvem** & 10 & 35 & 31 & 3,45 \\
Kappa no corte 0 - SAM & 0,164525 & 0,249362 & 0,377521 & 0,404186 \\
Kappa no corte 0 - SCM & 0,161943 & 0,243831 & 0,376299 & 0,399313 \\
\hline
\end{tabular}

* Número de imagens restantes no cubo após o corte. ** Máximo de porcentagem de cobertura de nuvem nas imagens do cubo resultante.

Org.: dos Autores, 2018. 
Dos três algoritmos o que obteve menor média do coeficiente Kappa foi o SCM $(0,411)$, na combinação 4C3AT (Tabela 3) O maior desvio padrão foi observado na mesma combinação, para os algoritmos SCM e SAM $(0,103)$. Os maiores coeficientes de variação são observados para os algoritmos SCM e SAM, sendo todos acima de $12 \%$. O menor coeficiente de variação é observado no algoritmo $\mathrm{MD}$, na combinação $2 \mathrm{C} 1 \mathrm{AT}$.

Tabela 3 - Análise das médias, desvio padrão e coeficiente de variação dos dados.

\begin{tabular}{cccccc}
\hline Algoritmo & Medidas & 4C.3AT & 4C.1AT & 2C.3AT & 2C.1AT \\
\hline \multirow{3}{*}{ MD } & Média & 0,498 & 0,498 & 0,573 & 0,747 \\
& Desvio padrão & 0,044 & 0,027 & 0,069 & 0,024 \\
& CV \% & 8,885 & 5,467 & 12,025 & 3,234 \\
\multirow{3}{*}{ SAM } & Média & 0,412 & 0,498 & 0,575 & 0,66 \\
& Desvio padrão & 0,103 & 0,093 & 0,088 & 0,085 \\
& CV \% & 25,09 & 18,77 & 15,24 & 12,94 \\
\multirow{3}{*}{ SCM } & Média & 0,411 & 0,498 & 0,575 & 0,66 \\
& Desvio padrão & 0,103 & 0,094 & 0,088 & 0,086 \\
& CV \% & 25,11 & 18,81 & 15,24 & 12,97 \\
\hline
\end{tabular}

Org.: dos Autores, 2018.

Na matriz de confusão do corte 80, algoritmo MD, 2 classes (Tabela 4) observa-se que nas combinações que utilizaram 3 assinaturas temporais, há maior confusão entre os pixels da classe "não floresta". Nas combinações com 4 classes (Tabela 5), observa-se menor confusão para as classes água, cana e floresta quando há aumento do número de amostras. Observa-se nos resultados das classificações que no corte 35 , houve grande confusão entre das classes com o pasto (Figura 6). 
Tabela 4. Matriz de confusão para as combinações com 2 classes, corte 80.

\begin{tabular}{ccccccc}
\hline Corte 80 & \multicolumn{3}{c}{ 2C.3AT } & \multicolumn{3}{c}{ 2C.1AT } \\
\hline MD & Não floresta & Floresta & Kappa & Não floresta & Floresta & Kappa \\
Não floresta & 20692 & 1475 & & 26966 & 3622 & 0.73 \\
Floresta & 12715 & 43518 & 0.61 & 6441 & 41371 & \\
SAM & Não floresta & Floresta & Kappa & Não floresta & Floresta & Kappa \\
Não floresta & 21575 & 1859 & \multirow{2}{*}{0.62} & 25332 & 3053 & \multirow{2}{*}{0.70} \\
Floresta & 11832 & 43134 & & 8075 & 41940 & \\
SCM & Não floresta & Floresta & Kappa & Não floresta & Floresta & Kappa \\
Não floresta & 21575 & 1859 & \multirow{2}{*}{0.62} & 25332 & 3054 & \multirow{2}{*}{0.70} \\
Floresta & 11832 & 43134 & & 8075 & 41939 & \\
\hline
\end{tabular}

Org.: dos Autores, 2018.

Tabela 5 - Matriz de confusão nas combinações de 4 classes, corte 80

\begin{tabular}{cccccccccccc}
\hline Corte 80 & \multicolumn{3}{c}{ 4C.3AT } & \multicolumn{1}{c}{ 4C.1AT } \\
\hline MD & Pasto & Água & Cana & Floresta & Kappa & Pasto & Água & Cana & Floresta & kappa \\
\hline Pasto & 16801 & 202 & 160 & 1038 & & 13075 & 106 & 126 & 2738 & \\
Água & 2814 & 181 & 34 & 963 & & 1714 & 177 & 51 & 431 & 0.47 \\
Cana & 2383 & 34 & 212 & 384 & 0.53 & 11253 & 151 & 249 & 424 & \\
Floresta & 10452 & 107 & 26 & 42609 & & 6408 & 90 & 6 & 41401 & \\
\hline SAM & Pasto & Água & Cana & Floresta & Kappa & Pasto & Água & Cana & Floresta kappa \\
\hline Pasto & 16046 & 140 & 98 & 1155 & & 18297 & 152 & 116 & 1809 & \\
Agua & 3976 & 263 & 89 & 1178 & & 3783 & 240 & 75 & 866 & 0.56 \\
Cana & 3469 & 36 & 145 & 978 & 0.51 & 3937 & 35 & 171 & 1132 & \\
Floresta & 8959 & 85 & 100 & 41683 & & 6433 & 97 & 70 & 41187 & \\
\hline SCM & Pasto & Água & Cana & Floresta & Kappa & Pasto & Água & Cana & Floresta & kappa \\
\hline Pasto & 16047 & 140 & 98 & 1155 & & 18299 & 152 & 116 & 1809 & \\
Agua & 3976 & 263 & 89 & 1178 & & 3782 & 240 & 75 & 866 & \\
Cana & 3469 & 36 & 145 & 978 & 0.51 & 3936 & 35 & 171 & 1132 & 0.56 \\
Floresta & 8958 & 85 & 100 & 41683 & & 6433 & 97 & 70 & 41187 & \\
\hline
\end{tabular}

Org.: dos Autores, 2018. 
Figura 6 - Resultado das classificações 2C3AT e 4C3AT (SAM e MD) corte 80.
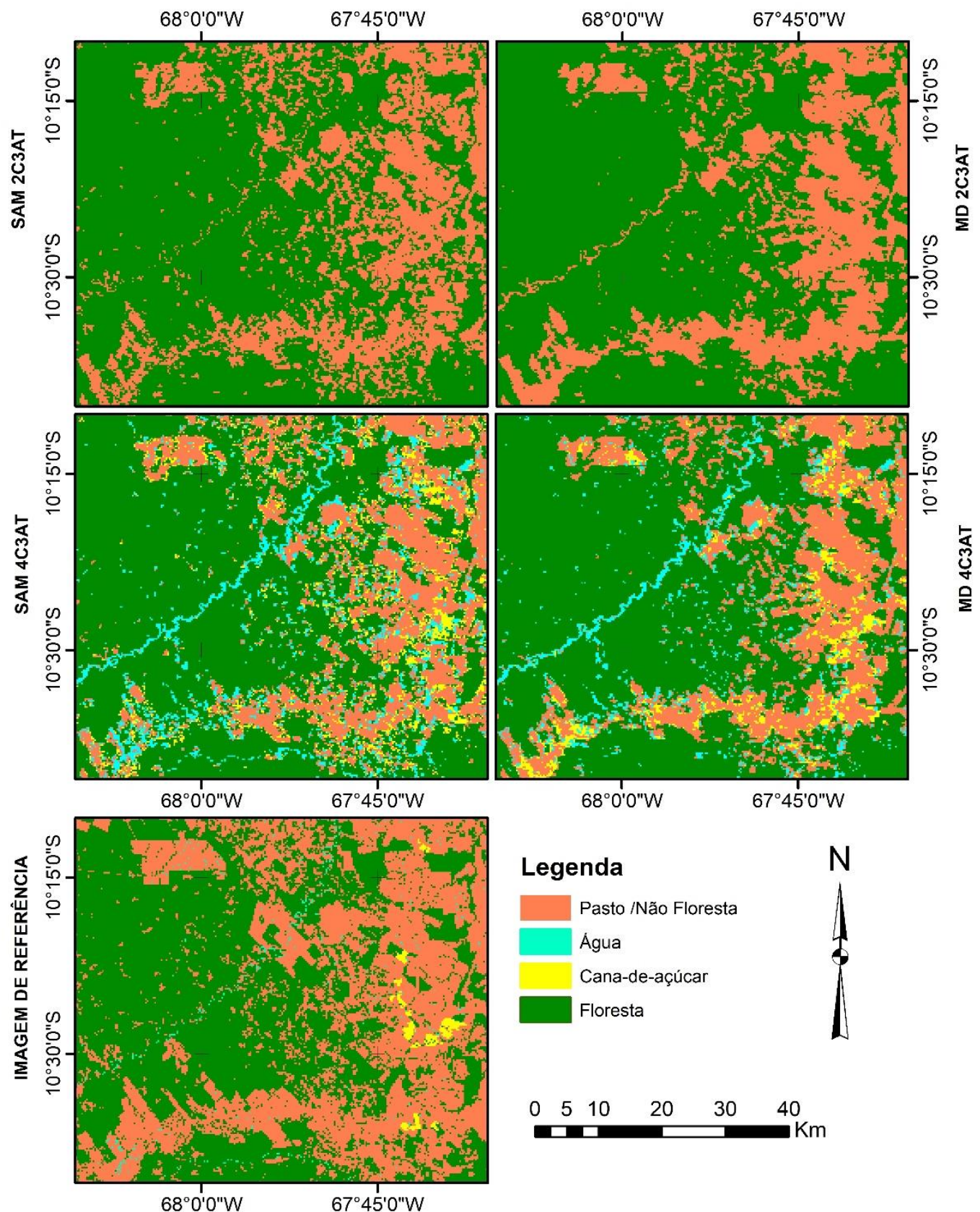

Org.: dos Autores, 2018.

\section{Discussão}

Nos testes realizados, foi possível observar que a manutenção das imagens com nuvem influencia o resultado das classificações. É perceptível 
que à medida em que essas imagens são retiradas, há uma melhora no resultado das classificações. No corte 0 , ou seja, na classificação do cubo com todas as imagens, encontrou-se os menores valores do coeficiente Kappa para todas as combinações, validando a forte influência da presença de nuvem nos resultados.

Para se atingir os melhores resultados nas classificações, os cubos mantiveram-se com 10 a 35 imagens de todo o período e a cobertura de nuvem foi mínima nesses cubos, variando de 0 a 5,91\%. Dentre os resultados, a combinação 4C1E usando o algoritmo MD apresentou uma exceção, sendo que o cubo de melhor coeficiente Kappa chegou a 59 imagens, com cobertura de nuvens variando de 0 a 53\%. Apesar de não ser um excelente coeficiente Kappa $(0,53)$, o resultado mostra que o algoritmo MD é menos sensível a presença de nuvens na série temporal. Outro dado que reforça esse fato é o baixo coeficiente de variação em todas as combinações em comparação com os outros algoritmos utilizados.

Os valores de coeficiente Kappa para os algoritmos testados variam com o número de classes de uso e cobertura da terra. Quando o número de classes aumenta, o coeficiente Kappa diminui corroborando com os resultados encontrados em outros trabalhos (ABADE et al., 2015). Nos testes com apenas duas classes (floresta e não floresta), os alvos possuem comportamentos temporais muito distintos, aumentando a eficiência dos algoritmos (SANTANA et al., 2010). Nos resultados para 4 classes, observou-se uma maior confusão dos alvos com redução do índice Kappa. As principais confusões ocorreram entre a classe cana-de-açúcar e pasto, fato observado em outros trabalhos com classificação de séries temporais envolvendo a cultura da cana (XAVIER et al., 2006; VICENTE et al., 2012). No caso do município de Capixaba, onde existem grandes áreas de pasto em contraste ao cultivo de cana, a confusão entre os alvos é comum (XAVIER et al., 2006).

Além disso, o processo de corte de imagens elimina principalmente as imagens do período chuvoso devido à maior cobertura de nuvem, aglutinando 
preferencialmente as imagens disponíveis na série temporal do período seco (junho a agosto). Portanto, a representação sazonal da fenologia vegetal podem ser prejudicadas com pouca representação do perído chuvoso. Somado a isso, a colheita da cana-de-acúcar ocorre nos perídos de junho e julho para a espécie cultivada no Estado do Acre (AMARAL et al., 2001; BARDALES et al., 2011). Assim, os perfis temporais de cana-de-acúcar podem ser confundidos com pasto ou capoeira (áreas desmatadas anteriormente e em estágio de regneração). Algumas áreas de cana tem seu período de colheita estendido para os meses posteriores a junho e julho, mantendo a cobertura vegetal durante o período de maior disponibilidade de imagens e tendendo a ter uma assinatura temporal semelhante às áreas de capoeira.

A identificação da água, apesar de ser um alvo claramente distinto dos demais, foi influenciada pela resolução especial do sensor. Os rios da área de estudo possuem uma extensão lateral estreita, que afeta a detecção de corpos nesta classe (ABADE et al., 2015).

Nos algoritmos SAM e SCM, o melhor coeficiente Kappa para as classificações que utilizaram 4 alvos foi de 0,59, mostrando a habilidade desses algoritmos na separação de alvos, mesmo em condições de alta presença de ruídos. Apesar desse coeficiente Kappa ser considerado um "bom" resultado, pode não atender às necessidades de estudos que exijam melhor acurácia.

Logo, o estudo de séries temporais na Amazônia é limitado pela presença de nuvens, sendo possível obter boas classificações, exigindo, no entanto, a retirada de grande parte das imagens da série temporal. Os resultados ficam prejudicados pela não possbilidade de acompanhamento da fenologia da cobertura vegetal ao longo de todo o ano, prejudicando este tipo de classificação. A alternativa para isto seria o uso de sensores que sofram menos influência das questões atmosféricas como, por exemplo, as imagens de radar. 


\section{Considerações Finais}

A presença de nuvem em séries temporais na Amazônia influenciou a qualidade das classificações. A quantidade de amostras utilizadas, o número de classes e os algoritmos de classificação tem influência sobre o número máximo de imagens utilizadas para garantir os melhores resultados. O algoritmo Mínima Distância (MD) destacou-se dentre os testes de 2 classes, atingindo um coeficiente Kappa considerado "muito bom". Para os testes com 4 classes, os algoritmos SAM e SCM tiveram melhor desempenho. Os melhores coeficientes Kappa foram encontrados em cubos temporais cujas imagens possuíam até $5 \%$ de cobertura de nuvem, sendo determinante para a escolha de imagens claras, livres de nuvens, para os estudos de séries temporais.

\section{Agradecimentos}

Os autores agradecem ao Programa de Pós-graduação em Geografia da Universidade de Brasília e ao Instituto Federal de Educacão, Ciência e Tecnologia de Brasília.

\section{Referências}

ABADE, N. A. et al. Comparative analysis of MODIS time-series classification using support vector machines and methods based upon distance and similarity measures in the brazilian cerrado-caatinga boundary. Remote Sensing, v. 7, n. 9, p. 12160-12191, 2015. https://doi.org/10.3390/rs70912160

ALI, A.; DE BIE, C. A. J. M.; SKIDMORE, A. K. Detecting long-duration cloud contamination in hyper-temporal NDVI imagery. International Journal of Applied Earth Observation and Geoinformation, v. 24, n. 1, p. 22-31, 2013. https://doi.org/10.1016/j.jag.2013.02.001 AMARAL, E. F. et al. Aptidão do solos do Acre para o cultivo da cana-de-açúcar. Comunidcado Técnica Embrapa, v. 143, p. 1-6, 2001.

ARTAXO, P. et al. Atmospheric aerosols in Amazonia and land use change: from natural biogenic to biomass burning conditions. Faraday Discussions, v. 165, n. 0, p. 203-235, 2013. https://doi.org/10.1039/c3fd00052d

ASNER, G. P. Cloud cover in Landsat observations of the Brazilian Amazon. International Journal of Remote Sensing, v. 22, n. 18, p. 3855-3862, 2001. https://doi.org/10.1080/01431160010006926 
BARDALES, N. G. et al. Zoneamento Agroclimático para Cultivo da Cana-de- açúcar em Três Municípios da Regional do Baixo Acre, Estado do Acre, Brasil. Rio Branco: [s.n.].

BRASWELL, B. H. et al. A multivariable approach for mapping sub-pixel land cover distributions using MISR and MODIS: Application in the Brazilian Amazon region. Remote Sensing of Environment, v. 87, n. 2-3, p. 243-256, 2003. https://doi.org/10.1016/j.rse.2003.06.002

CÂMARA, G. et al. Análise espacial e geoprocessamento. In: Gilberto Câmara; Clodoveu Davis; Antônio Miguel Vieira Monteiro (Org.). Introdução à ciência da geoinformação. São José dos Campos: INPE/DPI, 2002.

CHEN, G. et al. Spatiotemporal patterns of tropical deforestation and forest degradation in response to the operation of the Tucuruí hydroelectric dam in the Amazon basin. Applied Geography, v. 63, p. 1-8, 2015. https://doi.org/10.1016/j.apgeog.2015.06.001

CHEN, J. et al. A simple method for reconstructing a high-quality NDVI time-series data set based on the Savitzky-Golay filter. Remote Sensing of Environment, v. 91, n. 3-4, p. 332344, 2004. https://doi.org/10.1016/j.rse.2004.03.014

CONGALTONS, R. G. A Review of Assessing the Accuracy of Classifications of Remotely Sensed Data. Remote Sensing of Environment, p. 35-46, 1991. https://doi.org/10.1016/0034-4257(91)90048-B

DAAC, N. L. P.; FALLS, S. MODIS Land Products Quality Assurance Tutorial : Part-1 How to find, understand, and use the quality assurance information for MODIS land products. p. 1-17, 2014.

DE CARVALHO JUNIOR, O. A. et al. Classificação de padrões de savana usando assinaturas temporais ndvi do sensor modis no parque nacional chapada dos veadeiros. Revista Brasileira de Geofisica, v. 26, n. 4, p. 505-517, 2008. https://doi.org/10.1590/S0102261X2008000400010

DE CARVALHO JÚNIOR, O. A. et al. Combining Noise-Adjusted Principal Components transform and median filter techniques for denoising MODIS temporal signatures. Revista Brasileira de Geofisica, v. 30, n. 2, p. 147-157, 2012. https://doi.org/10.22564/rbgf.v30i2.88 FITZ, P. R. Geoprocessamento sem complicação. São Paulo: Oficina de Textos, 2008. FONSECA, L. M. G. Processamento digital de imagens. [s.l.] Instituto Nacional de Pesquisas Espaciais (INPE), 2000.

HILKER, T. et al. On the measurability of change in Amazon vegetation from MODIS. Remote Sensing of Environment, v. 166, p. 233-242, 2015. https://doi.org/10.1016/j.rse.2015.05.020

HUETE, A. et al. Overview of the radiometric and biophysical performance of the MODIS vegetation indices. Remote Sensing of Environment, v. 83, n. 1-2, p. 195-213, 2002. https://doi.org/10.1016/S0034-4257(02)00096-2

IBGE, I. B. DE G. E E. Produção Agrícola Municipal 2015Rio de JaneiroIBGE, 2016. Disponível em: <http://www.ibge.gov.br/estadosat/perfil.php?sigla=ac>

INPE. PRODES: Desflorestamento nos Municípios da Amazônia Legal para o ano de 2017Instituto Nacional de Pesquisas Espaciais (INPE), , 2017. Disponível em: $<$ http://www.dpi.inpe.br/prodesdigital/prodesmunicipal.php>

LANDIS, J. R.; KOCH, G. G. The Measurement of Observer Agreement for Categorical Data. Biometrics, v. 33, n. 1, p. 159-174, 1977. https://doi.org/10.2307/2529310

LATORRE, M. L. et al. Sensor Modis: Características Gerais e Aplicações. Espaço \& Geografia, v. 6, p. 91-121, 2003.

LI, L. et al. Monitoring the dynamics of surface water fraction from MODIS time series in a Mediterranean environment. International Journal of Applied Earth Observation and Geoinformation, v. 66, n. November 2017, p. 135-145, 2018. https://doi.org/10.1016/j.jag.2017.11.007

MARTINS, V. S. et al. Seasonal and interannual assessment of cloud cover and atmospheric constituents across the Amazon (2000-2015): Insights for remote sensing and climate 
analysis. ISPRS Journal of Photogrammetry and Remote Sensing, n. October 2017, p. 0-1, 2018. https://doi.org/10.1016/j.isprsjprs.2018.05.013

MASSEY, R. et al. MODIS phenology-derived, multi-year distribution of conterminous U.S. crop types. Remote Sensing of Environment, v. 198, p. 490-503, 2017. https://doi.org/10.1016/j.rse.2017.06.033

MELLO, M. P. et al. Spectral-temporal analysis by response surface applied to detect deforestation in the Brazilian Amazon. 2011 6th International Workshop on the Analysis of Multi-Temporal Remote Sensing Images, Multi-Temp 2011 Proceedings, n. 1, p. 89-92, 2011. https://doi.org/10.1109/Multi-Temp.2011.6005055

SANTANA, O. A. et al. Modelagem de espectros temporais NDVI-MODIS, no peŕiodo de 2000 a 2008, na bacia do Rio Paracatu, Brasil. Revista Brasileira de Geofisica, v. 28, n. 1, p. 47-60, 2010. https://doi.org/10.1590/S0102-261X2010000100004

SHELDON, S.; XIAO, X.; BIRADAR, C. Mapping evergreen forests in the Brazilian Amazon using MODIS and PALSAR 500-m mosaic imagery. ISPRS Journal of Photogrammetry and Remote Sensing, v. 74, p. 34-40, 2012. https://doi.org/10.1016/j.isprsiprs.2012.07.003 VERMOTE, E. F. MODIS Surface Reflectance User' s Guide. Orbit An International Journal On Orbital Disorders And Facial Reconstructive Surgery, p. 1-40, 2011.

VICENTE, L. E. et al. Séries temporais de NDVI do sensor SPOT Vegetation e algoritmo SAM aplicados ao mapeamento de cana-de-acçúcar. Pesquisa Agropecuaria Brasileira, v. 47, n. 9, p. 1337-1345, 2012. https://doi.org/10.1590/S0100-204X2012000900019

XAVIER, A. C. et al. Multi-temporal analysis of MODIS data to classify sugarcane crop. International Journal of Remote Sensing, v. 27, n. 4, p. 755-768, 2006. https://doi.org/10.1080/01431160500296735 